\title{
Precision medicine and personalising therapy in pulmonary hypertension: seeing the light from the dawn of a new era
}

\author{
Laurent Savale $e^{1,2,3}$, Christophe Guignabert ${ }^{1,3}$, Jason Weatherald ${ }^{4,5}$ and \\ Marc Humbert ${ }^{1,2,3}$
}

\author{
Number 7 in the Series "Personalised medicine in respiratory diseases" \\ Edited by Renaud Louis and Nicolas Roche
}

\begin{abstract}
Affiliations: ${ }^{1}$ Faculté de Médecine, Université Paris-Sud, Université Paris-Saclay, Le Kremlin-Bicêtre, France. ${ }^{2}$ AP-HP, Service de Pneumologie, Centre de Référence de l'Hypertension Pulmonaire Sévère, DHU Thorax Innovation, Hôpital de Bicêtre, Le Kremlin-Bicêtre, France. ${ }^{3}$ INSERM UMR_S 999, Le Plessis Robinson, France. ${ }^{4}$ Dept of Medicine, Division of Respirology, University of Calgary, Calgary, AB, Canada. ${ }^{5}$ Libin Cardiovascular Institute of Alberta, Calgary, AB, Canada.
\end{abstract}

Correspondence: Laurent Savale, Hôpital Bicêtre, Assistance Publique Hôpitaux de Paris, 78 rue du Général Leclerc, 94270 Le Kremlin Bicêtre, France. E-mail: laurent.savaledaphp.fr

@ERSpublications

Development of precision medicine strategies will be the next frontier in the evolution of PAH treatment http://ow.ly/8T8730j7e36

Cite this article as: Savale L, Guignabert C, Weatherald J, et al. Precision medicine and personalising therapy in pulmonary hypertension: seeing the light from the dawn of a new era. Eur Respir Rev 2018; 27: 180004 [https://doi.org/10.1183/16000617.0004-2018].

ABSTRACT Pulmonary hypertension $(\mathrm{PH})$ and pulmonary arterial hypertension $(\mathrm{PAH})$ include different cardiopulmonary disorders in which the interaction of multiple genes with environmental and behavioural factors modulates the onset and the progression of these severe conditions. Although the development of therapeutic agents that modulate abnormalities in three major pathobiological pathways for PAH has revolutionised our approach to the treatment of $\mathrm{PAH}$, the long-term survival rate remains unsatisfactory. Accumulating evidence has underlined that clinical outcomes and responses to therapy in PAH are modified by multiple factors, including genetic variations, which will be different for each individual. Since precision medicine, also known as stratified medicine or personalised medicine, aims to better target intervention to the individual while maximising benefit and minimising harm, it has significant potential advantages. This article aims to assemble and discuss the different initiatives that are currently underway in the $\mathrm{PH} / \mathrm{PAH}$ fields together with the opportunities and prospects for their use in the near future.

Previous articles in this series: No. 1: Chung KF. Personalised medicine in asthma: time for action. Eur Respir Rev 2017; 26: 170064. No. 2: Bonsignore MR, Suarez Giron MC, Marrone O, et al. Personalised medicine in sleep respiratory disorders: focus on obstructive sleep apnoea diagnosis and treatment. Eur Respir Rev 2017; 26: 170069. No. 3: Mascaux C, Tomasini P, Greillier L, et al. Personalised medicine for nonsmall cell lung cancer. Eur Respir Rev 2017; 26: 170066. No. 4: Noell G, Faner R, Agusti A. From systems biology to P4 medicine: applications in respiratory medicine. Eur Respir Rev 2018; 27: 170110. No. 5: Wouters EFM, Wouters BBREF, Augustin IL, et al. Personalised pulmonary rehabilitation in COPD. Eur Respir Rev 2018; 27: 170125. No. 6: Kokosi MA, Margaritopoulos GA, Wells AU. Personalised medicine in interstitial lung diseases. Eur Respir Rev 2018; 27: 170117.

Received: Jan 152018 | Accepted after revision: March 212018

Provenance: Commissioned article, peer reviewed.

Copyright OERS 2018. ERR articles are open access and distributed under the terms of the Creative Commons Attribution Non-Commercial Licence 4.0. 


\section{Introduction}

Pulmonary hypertension $(\mathrm{PH})$ refers to a haemodynamic and pathophysiological condition that is defined as a persistent elevation of the mean pulmonary arterial pressure $\geqslant 25 \mathrm{mmHg}$ at rest confirmed by right heart catheterisation, which leads to right ventricular failure and premature death. The symptoms of $\mathrm{PH}$ are nonspecific and include breathlessness, fatigue, weakness, angina and syncope and take years to appear, usually after the $\mathrm{PH}$ has reached an advanced stage. $\mathrm{PH}$ can result from pre-capillary (arterial) or post-capillary (venous) pathogenic mechanisms and occurs in a large group of diseases $[1,2]$.

Since the second world symposium on PH held in Evian in 1998, a clinical classification was established using a precision medicine approach in order to individualise and gather together different categories of $\mathrm{PH}$ sharing similar haemodynamic criteria, types of pulmonary vascular lesions and similar management $[1,2]$. Indeed, the current clinical classification for $\mathrm{PH}$ attempts to reconcile pathological findings with new pathobiological concepts and separates conditions with a predominantly pulmonary arterial disease component (group 1, also known as pulmonary arterial hypertension (PAH)) from four other disease categories composed of $\mathrm{PH}$ associated with left heart disease (group 2); $\mathrm{PH}$ associated with lung diseases and hypoxia (group 3); $\mathrm{PH}$ due to chronic thromboembolism (group 4); and $\mathrm{PH}$ due to unclear multifactorial mechanisms (group 5).

This clinical PH classification tool is well accepted and widely used in the daily practice of PH experts, allowing not only a more rapid introduction of targeted treatment avoiding harm and increasing benefit, but also facilitating outcome prediction and research strategies. However, this "one size fits all" approach yields an average treatment effect for the average patient, but is suboptimal for choosing therapy at the individual patient level. Within a clinical trial, there are probably certain groups of patients who respond very well to a treatment and groups of patients who respond poorly or who are made worse. The next step would be to include biomarkers, proteomics and genomics to further develop precision medicine strategies leading to personalised medicine for $\mathrm{PH}$ patients; however, several challenges remain. The objective of this review is to summarise some of the most common challenges and provides a brief overview on the existing literature.

\section{How could precision medicine add to current PAH/PH management?}

The goal of personalised medicine is to streamline clinical decision to provide the right treatment strategy for a given patient based on his or her individual characteristics (figure 1) [3]. It consists of classifying each patient into a subpopulation, taking into account their susceptibility to develop a particular disease or phenotype and its response to a treatment. Precision medicine, which is more ambitious, encompasses both the treatment and the prevention of the disease based on individual genetic, environmental and lifestyle differences. This new approach depends on the availability of molecular profiling tests for a particular clinical condition. The development of targeted therapies and immunotherapy in the treatment of cancer demonstrates that precision medicine is realisable. This new approach has allowed the possibility of more rapid introduction of cancer drugs and treatments according to a cancer's molecular profile regardless of location or histological analysis.

For patients with idiopathic/heritable $\mathrm{PAH}$, the 5- and 7-year survival rates from time of diagnostic right-sided heart catheterisation are $57 \%$ and $49 \%$, respectively, despite currently available medical treatment advances [4]. PAH is not a cancer, but similarities can be observed concerning the altered crosstalk between cells from different tissue types, unexplained proliferation and survival of pulmonary smooth muscle and endothelial cells, the metabolic (glycolytic) shifts and the association with the immune system [5]. PAH is characterised by vasoconstriction, cell accumulation in the vascular wall and intimal thickening of the small- to medium-sized pulmonary arteries $(\leqslant 500 \mu \mathrm{m})$ in the absence of major pulmonary parenchymal changes or embolic events [5-9]. Current PAH-specific therapies mainly induce relaxation of the pulmonary arteries. In a small group of PAH patients that respond to acute vasodilators, relaxation of pulmonary vascular smooth muscle can be achieved with calcium channel blockers. However, most patients are treated with specific medication approved by the United States Food and Drug Administration (US FDA) to modulate abnormalities in three major pathobiological pathways for PAH: the nitric oxide, prostacyclin and endothelin pathways [2]. Lung transplantation is the last therapeutic option if the disease progresses or medications fail.

Although different forms of $\mathrm{PAH} / \mathrm{PH}$ could reflect distinct pathophysiological mechanisms, current evidence strongly suggests that a common denominator underlying many of the established molecular and cellular elements exists [5-9]. Indeed, recent discoveries support the concept that this irreversible pulmonary vascular remodelling in $\mathrm{PAH}$ results from two complementary mechanisms: inherent characteristics of resident pulmonary vascular cells, including pulmonary artery smooth muscle cells (SMCs), myofibroblasts, endothelial cells and pericytes; and dysregulation of molecular events that govern pulmonary vascular cell growth/survival, including signals originating from microenvironmental alterations in the pulmonary arterial wall. Although tremendous advances have been made, the 


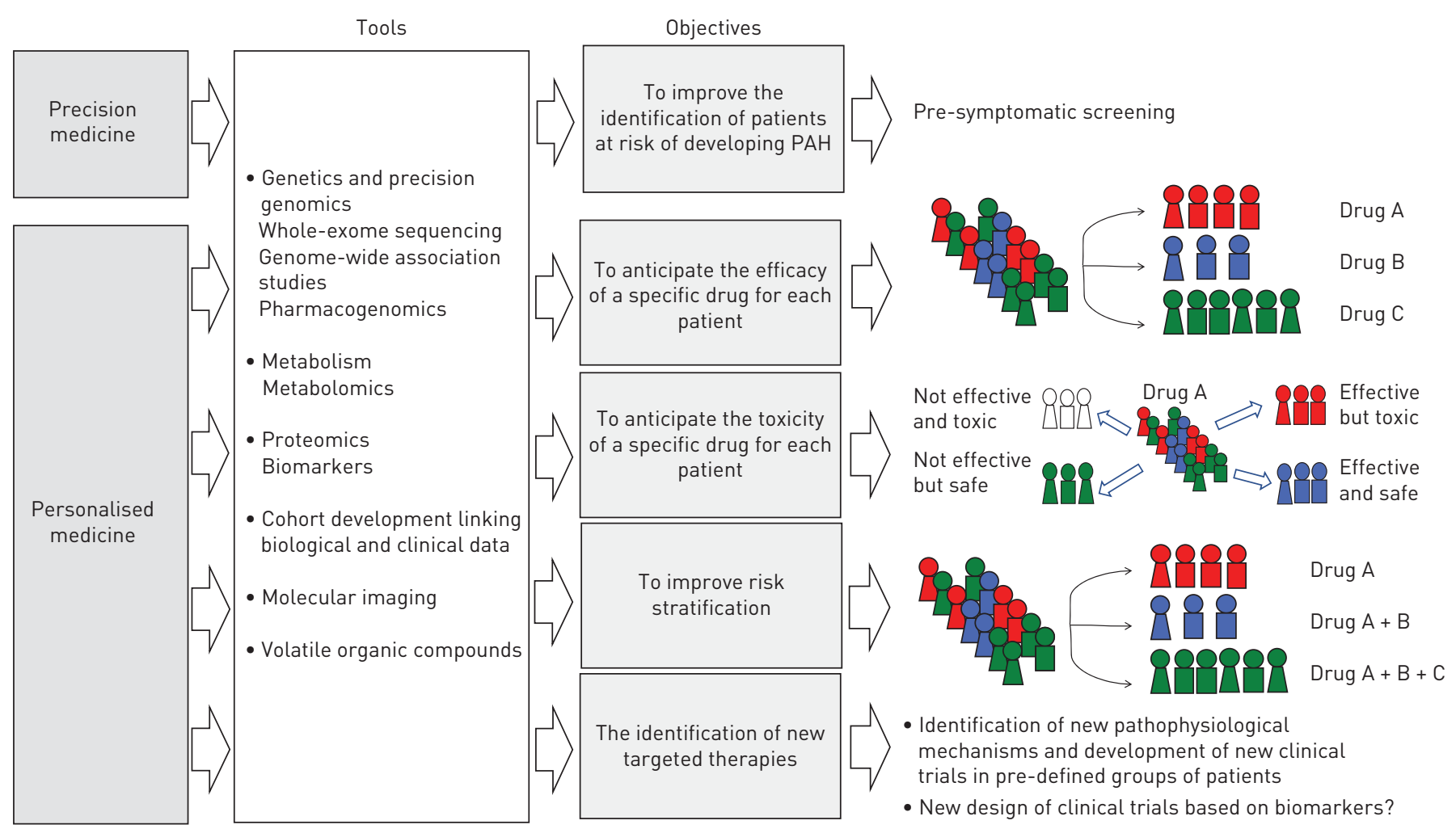

FIGURE 1 New “-omics" approach for personalised medicine. PAH: pulmonary arterial hypertension. Reproduced and modified from [3] with permission.

pathobiology remains poorly understood. Furthermore, it is now well established that the presence of genetic or environmental modifying factors can influence the onset and the progression of these severe cardiopulmonary disorders.

When considering idiopathic PAH patients, we are now beginning to appreciate a high degree of phenotypic variability within this subgroup. In the historical cohort from the National Institute of Health, patients with idiopathic PAH were young (mean age 31 years) with a female predominance [10]. Since then, idiopathic $\mathrm{PAH}$ has been increasingly diagnosed in older patients with a median age at diagnosis that continues to climb in national and international registries. A significant proportion of patients now present with other comorbidities that may influence prognosis, progression of disease and, probably, response to treatment $[11,12]$. Current guideline recommendations only take into account the clinical classification of $\mathrm{PH}$ and disease severity at diagnosis and during follow-up, resulting in highly variable treatment responses from one individual to the next. Therefore, it seems necessary to develop better methods of predicting responses to each therapeutic class of medication and to anticipate disease evolution at the individual level to optimise prognosis through individualised therapeutic choices. In addition, this high degree of phenotypic variability between the different $\mathrm{PH}$ entities or even within the same subgroup is a source of patient heterogeneity in clinical trials.

New "-omics" approaches permit the acquisition of large amounts of data at multiple biological levels, from gene sequencing to protein expression and metabolic profiling. Such data can cover all potential mechanisms implicated in underlying cellular networks that influence the function of organ systems in their totality [13]. This inversion of the classical research model (from traditional experimentation based on precise hypotheses to a model of research that does not depend at all on such hypotheses) opens the door in an unprecedented way to many new discoveries on the pathophysiological mechanisms in PAH, as well as to a better understanding of the molecular factors that influence the efficacy of existing therapies and prognosis. Therefore, our current approach must evolve urgently. The new era will be one not based on pre-specified hypotheses stemming from animal models, but one based on the identification of new pathophysiological mechanisms that are actually implicated in $\mathrm{PAH}$ in humans, using large-scale analyses of biological, molecular and genetic variations associated with the development of PAH. This new approach necessitates a reorganisation of research methods. Henceforth, it will be essential to establish biobanks from cohorts included in registries or clinical trials and to develop tissue banks from explanted lungs from patients who undergo lung transplantation. These new research perspectives will allow 
comparisons of patient characteristics, prognosis and responses to different classes of medications according to their epigenetic, genetic, proteomic and metabolomic signatures. We can hope that, sooner rather than later, clinicians will be able to establish a sort of individual "-omics map" to accurately anticipate the risk of progression and choose the most appropriate and effective therapy for each patient and improve outcomes. Importantly, the use of clinical phenotype and classification tools could help to minimise patient heterogeneity in $\mathrm{PH}$ clinical trials.

\section{What types of molecular testing could be used?}

Considerable progress has been achieved in oncology through the comprehensive assessment of the molecular features of patients and their therapeutic needs. In addition to the availability of clinical trials or compassionate use of specific therapeutic agents, these achievements typically relied on assays of blood and tissue that allow access to tumour specimen characterisation, well-validated circulating biomarkers and molecular imaging, among others. In PAH, tissue access is a clear limitation, since lung biopsy carries prohibitive risks in most $\mathrm{PAH}$ patients. Furthermore, the recent emergence and application of a large number of other new technologies in the field may lead to a precision medicine paradigm in the near future.

Genomics approaches have successfully identified several genes and genetic loci involved in the predisposition or in the development of $\mathrm{PH} / \mathrm{PAH}$ [14-41]. These findings have revealed complex multifactorial aspects of the $\mathrm{PH} / \mathrm{PAH}$ pathogenesis and have provided insights for disease diagnosis, treatment and prevention. Additionally, other omics-based technologies have been used in preclinical research to evaluate changes in other biomolecules, such as epigenomics for epigenetic markers, transcriptomics for mRNAs, non-coding RNAs and small RNAs, proteomics for proteins and peptides and metabolomics for low molecular weight metabolites. The utility of molecular classification to support clinical classification has been recently developed [42-44] and should foster the use of these technologies in the future. Indeed, Hemnes et al. [43] identified several rare gene variants involved in the acute vasodilator response. Another example of how pharmacogenomics can be used to personalise PAH treatment with currently available drugs comes from BENZA et al. [45] who used genome-wide association studies to identify single-nucleotide polymorphisms in the endothelin-1 gene, endothelin receptor $\mathrm{A}$ and $\mathrm{B}$ genes and G-protein subunit genes. They identified a variant in the GNG2 gene on chromosome 24 that was associated with better response to endothelin receptor antagonist therapy. Identification of favourable and detrimental polymorphisms in other PAH pathways, including the nitric oxide and prostacyclin pathways, may improve efficiency and selection of patients for future studies of existing or novel PAH therapies, including gene editing, gene replacement and endothelial progenitor cell therapies $[46,47]$.

There is a clear need to identify well-validated circulating or molecular imaging biomarkers in PH/PAH. In this context, RHODES et al. [44] performed a proteomic analysis in 143 consecutive patients with idiopathic and heritable PAH with 2 years of follow-up and in a further 75 patients with 2.5 years of follow-up. They identified that the measurements of a combination of nine circulating proteins can be used to stratify PAH patients with a high risk of mortality, independent of existing clinical assessments, which might have a use in clinical management and the evaluation of new therapies. This panel of nine prognostic proteins has been identified using an aptamer-based assay of 1129 plasma proteins and includes interleukin-1 receptor-like 1, tissue inhibitors of metalloproteinases-1 and -2, plasminogen, apolipoprotein-E, erythropoietin, complement factors $\mathrm{H}$ and $\mathrm{D}$ and insulin-like growth factor binding protein-1. Interestingly, the functions of these proteins were related to myocardial stress, inflammation, pulmonary vascular cellular dysfunction and structural dysregulation, iron status and coagulation. In addition to genomics and metabolomics, these circulating biomarkers could clearly help to obtain a better comprehensive assessment of the molecular features of $\mathrm{PH} / \mathrm{PAH}$ patients and therefore needs to be validated.

Other techniques are in development with the objective of screening and early detection of PAH at the individual level. For example, volatile organic compounds (VOCs) have been proposed as noninvasive biomarkers for PAH and an "artificial nose" has been developed with the ability to analyse the pattern of exhaled VOCs unique to an individual. Pathological processes affect the production and may drive specific profiles of VOCs in the exhaled breath, known as the "exhaled volatolome". In this way, the identification and quantification of an exhaled volatolome could provide important, personalised, noninvasive biomarker information [48].

POLLETT et al. [49] reported a new technique to harvest pulmonary arterial endothelial cells (PAECs) from discarded Swan-Ganz catheter balloons after routine right heart catheterisation. The major advancement of this new technology is the ability to isolate PAECs from a living human rather than from explanted or post mortem tissue. This approach could have the potential for future applications to test pharmaceutical responses in vitro for individual patients and to provide a direct examination of changes in endothelial 
biology during disease progression and in response to treatment $[50,51]$. The use of induced pluripotent stem cell (iPSCs) to derive endothelial or smooth muscle cells from PAH patient could represent an alternative and a path to move PAH treatment into personalised medicine $[52,53]$.

\section{Is precision medicine ready for use in $\mathrm{PAH} / \mathrm{PH}$ ?}

The practice of precision medicine is not really new in $\mathrm{PH} / \mathrm{PAH}$. For decades, pulmonary vasodilator testing to inhaled nitric oxide has been used to guide management of patients with PAH. Patients meeting vasoreactive criteria during right heart catheterisation are known to have favourable long-term responses to oral calcium channel blockers and better survival [54, 55]. Although vasodilator-responsive PAH patients are a minority $(<10 \%)$ of idiopathic PAH patients, recent studies have found distinct genetic and molecular pathways in vasodilator-responsive patients compared to nonvasoresponsive PAH [43, 56], supporting the utility of molecular techniques in predicting drug responsiveness in $\mathrm{PH} / \mathrm{PAH}$.

While precision medicine has the potential to profoundly improve the $\mathrm{PH} / \mathrm{PAH}$ management, different challenges must be resolved to extend precision medicine approaches into mainstream practice. First, a better understanding of how altered immune responses may contribute to $\mathrm{PAH}$ initiation, perpetuation and worsening is needed. It is now well established that altered inflammatory and immune mechanisms play a significant role in $\mathrm{PH} / \mathrm{PAH}$ by recruiting inflammatory cells, remodelling the pulmonary vasculature and promoting autoimmune responses. Indeed, there is complex interplay between PAH and autoimmune disorders (such as systemic lupus erythematosus, Sjögren's syndrome, scleroderma and thyroiditis) or chronic infections (HIV, bilharzia and human herpes virus type 8). Some of these clinical presentations may benefit from specific management, leading to a distinct outcome from idiopathic PAH. For example, pulmonary vascular disease may be reversible with immunosuppressant medications in some patients with lupus or mixed connective tissue disease-associated $\mathrm{PAH}$, whereas this type of response to immunosuppressive medications is not observed in systemic sclerosis-associated PAH [57]. An unusual response to specific PAH therapies (including endothelin receptor antagonists) has also been observed in particular forms of PAH that are associated with immune disorders and an inflammatory component that probably plays a crucial role. Thus, cases of normalisation or quasi-normalisation of resting haemodynamics have been reported in patients with HIV or cirrhosis $[58,59]$. More generally, inflammation and immune disorders appear to be common denominators for all forms of PAH. In PAH, pulmonary vascular lesions are characterised by varying degrees of perivascular inflammatory infiltrates, comprising of $\mathrm{T}$ - and B-lymphocytes, macrophages, dendritic cells and mast cells. Furthermore, PAH is associated with increased circulating and lung levels of certain cytokines, chemokines and adipokines [8]. Interestingly, some of these circulating inflammatory mediators correlate with a worse clinical outcome in PAH patients [60, 61]. In addition, circulating anti-endothelial cells and antifibroblast antibodies are found in $10-40 \%$ of patients suffering from idiopathic PAH [62-65], which may indicate the presence of autoimmune mechanisms linked to complement activation in the genesis of pulmonary vascular lesions of PAH. Consistently, altered regulatory T-cell function [66, 67], T-helper 17 cell immune polarisation [68], dendritic cell recruitment in pulmonary vascular lesions [69] and the presence of lymphoid neogenesis in lungs have been demonstrated in patients with $\mathrm{PAH}[70,71]$. However, the fact that steroid or aspirin treatment are clearly not effective in idiopathic and heritable PAH [72], and that prostacyclin, which has anti-inflammatory properties [73-75], does not reverse the pulmonary vascular remodelling and $\mathrm{PAH}$, support the need for a better characterisation of the involvement and mechanism of distinct immune cells and key inflammatory mediators. This better knowledge could pave the way to novel therapeutic strategies for predefined groups of patients and could help to identify panels of key inflammatory mediators to distinguish different $\mathrm{PH} / \mathrm{PAH}$ clinical phenotypes.

Second, the reasons why bone morphogenetic protein receptor (BMPR)-II signalling is reduced in PAH and how the alterations influence or even drive the pathogenesis need to be understood. Indeed, heterozygous BMPR2 germline mutations account for more than $75-80 \%$ of familial PAH and $\sim 15-20 \%$ of sporadic cases. However, the average penetrance of BMPR2 pathogenic variants is low and the lifetime risk of developing PAH with a BMPR2 pathogenic variation in a male is $14 \%$, whereas in a female it is $42 \%$ [38]. Because there is evidence that BMPR2 mutation carriers have a worse prognosis [38], are less likely to respond acutely to vasodilators and have a relatively preserved diffusing capacity of the lung for carbon monoxide compared to non-carriers [76, 77], a systematic screening for the presence of this predisposing mutation is recommended. Evidence suggests that the degree of vascular remodelling is greater in lungs of patients with BMPR2 mutations compared with lungs of non-BMPR2 related disease at the time of transplantation [78, 79]. Interestingly, for an unknown reason, BMPR-II or its downstream signalling pathways are reduced to a similar extent in explanted lungs of non-carriers, highlighting the need of further studies to identify the underlying mechanisms [80]. Additional mutations in several other genes ( $>1 \%$ of families with $\mathrm{PAH}$ ) have been identified, namely ACVRL1 (activin receptor-like kinase (ALK-1), BMPR1B (ALK-6), GDF2 (bone morphogenetic protein-9), TBX4 (T-box 4), ENG (endoglin), 
SMAD9 (Smad-8), CAV (caveolin) and KCNK3 [81]. The development of genetic counselling and the analysis of the phenotypic profile of patients according to their genotype have helped to develop preventive medicine and a specific monitoring for BMPR2 mutation carriers. Therefore, pre-symptomatic screening of individuals with known mutations in PAH-predisposing genes could potentially lead to earlier diagnosis and treatment of PAH. Considering the above, the 2015 European Society of Cardiology/ European Respiratory Society guidelines currently recommend that "individuals who test positive for $\mathrm{PAH}$-causing mutations and first degree relatives of heritable $\mathrm{PAH}$ cases may be considered to have an annual screening echocardiogram (IIb-C)". The clinical presentation of heritable PAH at diagnosis and its evolution can be influenced by the identified genetic mutation leading in some cases to a personalisation of therapeutic choices and monitoring. Patients with established PAH and a BMPR2 mutation have an increased risk of death or transplantation [38]. In addition, biallelic EIF2AK4 mutations, known to associate with pulmonary veno-occlusive disease and/or pulmonary capillary haemangiomatosis have been recently discovered [82] allowing appropriate management and early referral for lung transplantation.

Thus, not all carriers of a BMPR2 mutation will develop PAH, suggesting that other factors, genetic and/or environmental, are required to induce PAH. While causal in disease pathogenesis, the exact mechanism between BMPR-II dysfunction and the development of PAH is not known. Even in the absence of a mutation, BMPR-II expression is reduced in PAH [83]; therefore, improving BMPR-II signalling could be applicable beyond those with heritable or sporadic BMPR2 mutations. A genomics-driven classification with therapeutic strategies targeting the BMPR-II pathway is an attractive paradigm for precision medicine in PAH patients.

Gene editing and gene therapy are promising emerging technologies that could correct pathogenic mutations in patients with heritable PAH [3]. Using an adenovirus vector, REYNOLDs et al. [84] showed that BMPR2 gene delivery could restore BMPR-II expression, improve haemodynamics and attenuate vascular remodelling in monocrotaline-induced and chronic-hypoxia models of $\mathrm{PH}$ in rats. More recent studies have confirmed the ability of BMPR2 gene therapy to improve PAH in animal models by increasing Smad1/5/8 signalling and possibly by increasing nitric oxide production in human pulmonary endothelial cells [85]. YAN et al. [86] showed that myeloablative radiation followed by transplantation of donor stem cells from mice with normal $B M P R 2$ into mice with BMPR2 mutations prevented the development of $\mathrm{PH}$. Although these pre-clinical data are promising, there are likely to be significant challenges in translating $B M P R 2$ gene therapy to humans with our current limitations in vector delivery to the pulmonary endothelium, immunogenicity and uncertainty regarding whether increasing BMPR-II expression results in sustainable long-term improvements in $\mathrm{PAH}$.

Other methods of restoring BMPR-II expression and function have been sought. SPIEKERKOETTER et al. [87] used a high-throughput transcriptional assay to screen 3756 compounds that were already approved by the US FDA for BMPR-II activation. They found that tacrolimus (FK506), a calcineurin inhibitor commonly used as an immunosuppressant, activates the BMPR-II pathway even in the absence of functional BMPR-II receptor. In addition, tacrolimus prevented and even reversed $\mathrm{PH}$ in rodent monocrotaline and Sugen-hypoxia models. In a subsequent phase IIa study, they reported that low-dose FK506 improved BMPR-II signalling in three patients with end-stage PAH without BMPR2 mutations, with concurrent clinical improvement [88]. In a recent double-blind placebo-controlled randomised study, SPIEKERKOETTER et al. [89] used low dose FK506 in New York Heart Association functional class II and III PAH patients. In the 23 patients randomised, BMPR-II expression was compared to healthy controls and FK506 was well tolerated overall; however, there were no significant improvements in clinical variables such as 6-min walking distance, N-terminal pro-brain natriuretic peptide, echocardiographic measures of right ventricular function or BMPR-II expression compared to placebo [89]. There have been no studies or reports of FK506 used in patients with heritable PAH due to BMPR2 mutations, specifically. Two other pre-clinical studies have shown that chloroquine can improve BMPR-II signalling by inhibiting lysosomal degradation of BMPR-II, which prevents proliferation of pulmonary artery SMCs and progression of $\mathrm{PH}[90,91]$.

Finally, other challenges such as data quality/integrity, reproducibility, security and study sample sizes have to be addressed. The small number of multi-omics datasets and the lack of standardised and harmonised protocols is also a limitation that affects the dissemination of these approaches.

\section{Conclusion}

Enthusiasm for the prospect of precision medicine has grown significantly in the last few years. However, the $\mathrm{PH} / \mathrm{PAH}$ field already has several emerging clinically actionable tools useful for precision medicine and even if the translation of these technologies into clinically tools is slow, their usages will probably increase in the near future. 
Author contributions: All the authors wrote and edited the manuscript.

Conflict of interest: L. Savale reports grants and personal fees from Actelion and Bayer, and personal fees from MSD and GSK, during the conduct of the study. J. Weatherald reports grants from the European Respiratory Society and the Canadian Thoracic Society, as well as personal fees and non-financial support from Actelion and Bayer, outside the submitted work. M. Humbert reports personal fees from Actelion Pharmaceuticals Ltd, United Therapeutics and Pfizer, and grants and personal fees from Bayer, during the conduct of the study. He has received personal fees from Novartis, outside the submitted work. In addition to being investigator in trials involving these companies, relationships include consultancy service and membership of scientific advisory boards.

\section{References}

1 Simonneau G, Gatzoulis MA, Adatia I, et al. Updated clinical classification of pulmonary hypertension. J Am Coll Cardiol. 2013; 62: 25 Suppl, D34-D41.

2 Galie N, Humbert M, Vachiery J-L, et al. 2015 ESC/ERS Guidelines for the diagnosis and treatment of pulmonary hypertension. The Joint Task Force for the Diagnosis and Treatment of Pulmonary Hypertension of the European Society of Cardiology (ESC) and the European Respiratory Society (ERS). Eur Respir J 2015; 46: 903-975.

3 Austin ED, West J, Loyd JE, et al. Translational advances in the field of pulmonary hypertension molecular medicine of pulmonary arterial hypertension. From population genetics to precision medicine and gene editing. Am J Respir Crit Care Med 2017; 195: 23-31.

4 Benza RL, Miller DP, Barst RJ, et al. An evaluation of long-term survival from time of diagnosis in pulmonary arterial hypertension from the REVEAL Registry. Chest 2012; 142: 448-456.

5 Guignabert C, Tu L, Le Hiress M, et al. Pathogenesis of pulmonary arterial hypertension: lessons from cancer. Eur Respir Rev 2013; 22: 543-551.

6 Guignabert C, Dorfmuller P. Pathology and pathobiology of pulmonary hypertension. Semin Respir Crit Care Med 2013; 34: 551-559.

7 Guignabert C, Tu L, Girerd B, et al. New molecular targets of pulmonary vascular remodeling in pulmonary arterial hypertension: importance of endothelial communication. Chest 2015; 147: 529-537.

8 Guignabert C, Dorfmuller P. Pathology and pathobiology of pulmonary hypertension. Semin Respir Crit Care Med 2017; 38: 571-584

9 Huertas $\mathrm{A}, \mathrm{Tu} \mathrm{L}$, Guignabert C. New targets for pulmonary arterial hypertension: going beyond the currently targeted three pathways. Curr Opin Pulm Med 2017; 23: 377-385.

10 D'Alonzo GE, Barst RJ, Ayres SM, et al. Survival in patients with primary pulmonary hypertension. Results from a national prospective registry. Ann Intern Med 1991; 115: 343-349.

11 Humbert M, Sitbon O, Chaouat A, et al. Survival in patients with idiopathic, familial, and anorexigen-associated pulmonary arterial hypertension in the modern management era. Circulation 2010; 122: 156-163.

12 Ling Y, Johnson MK, Kiely DG, et al. Changing demographics, epidemiology, and survival of incident pulmonary arterial hypertension: results from the pulmonary hypertension registry of the United Kingdom and Ireland. Am J Respir Crit Care Med 2012; 186: 790-796.

13 Nicholson JK, Lindon JC. Systems biology: metabonomics. Nature 2008; 455: 1054-1056.

14 Germain M, Eyries M, Montani D, et al. Genome-wide association analysis identifies a susceptibility locus for pulmonary arterial hypertension. Nat Genet 2013; 45: 518-521.

15 Vattulainen S, Aho J, Salmenperä $\mathrm{P}$, et al. Accurate genetic diagnosis of Finnish pulmonary arterial hypertension patients using oligonucleotide-selective sequencing. Mol Genet Genomic Med 2015; 3: 354-362.

16 Austin ED, Ma L, LeDuc C, et al. Whole exome sequencing to identify a novel gene (caveolin-1) associated with human pulmonary arterial hypertension. Circ Cardiovasc Genet 2012; 5: 336-343.

17 Gómez J, Reguero JR, Alvarez C, et al. A semiconductor chip-based next generation sequencing procedure for the main pulmonary hypertension genes. Lung 2015; 193: 571-574.

18 Aldred MA, Comhair SA, Varella-Garcia M, et al. Somatic chromosome abnormalities in the lungs of patients with pulmonary arterial hypertension. Am J Respir Crit Care Med 2010; 182: 1153-1160.

19 Yeager ME, Halley GR, Golpon HA, et al. Microsatellite instability of endothelial cell growth and apoptosis genes within plexiform lesions in primary pulmonary hypertension. Circ Res 2001; 88: E2-E11.

20 Machado RD, James V, Southwood M, et al. Investigation of second genetic hits at the BMPR2 locus as a modulator of disease progression in familial pulmonary arterial hypertension. Circulation 2005; 111: 607-613.

21 Kataoka M, Aimi Y, Yanagisawa R, et al. Alu-mediated nonallelic homologous and nonhomologous recombination in the BMPR2 gene in heritable pulmonary arterial hypertension. Genet Med 2013; 15: 941-947.

22 Mutlu Z, Kayıkçioğlu M, Nalbantgil S, et al. Sequencing of mutations in the serine/threonine kinase domain of the bone morphogenetic protein receptor type 2 gene causing pulmonary arterial hypertension. Anatol J Cardiol 2016; 16: 491-496.

23 Deng Z, Morse JH, Slager SL, et al. Familial primary pulmonary hypertension (gene PPH1) is caused by mutations in the bone morphogenetic protein receptor-II gene. Am J Hum Genet 2000; 67: 737-744.

24 Lane KB, Machado RD, Pauciulo MW, et al. Heterozygous germline mutations in BMPR2, encoding a TGF- $\beta$ receptor, cause familial primary pulmonary hypertension. Nat Genet 2000; 26: 81-84.

25 Chaouat A, Coulet F, Favre C, et al. Endoglin germline mutation in a patient with hereditary haemorrhagic telangiectasia and dexfenfluramine associated pulmonary arterial hypertension. Thorax 2004; 59: 446-448.

26 Harrison RE, Berger R, Haworth SG, et al. Transforming growth factor- $\beta$ receptor mutations and pulmonary arterial hypertension in childhood. Circulation 2005; 111: 435-441.

27 Abdalla SA, Gallione CJ, Barst RJ, et al. Primary pulmonary hypertension in families with hereditary haemorrhagic telangiectasia. Eur Respir J 2004; 23: 373-377.

28 Ma L, Roman-Campos D, Austin ED, et al. A novel channelopathy in pulmonary arterial hypertension. $N$ Engl Med 2013; 369: 351-361.

29 Shintani M, Yagi H, Nakayama T, et al. A new nonsense mutation of SMAD8 associated with pulmonary arterial hypertension. J Med Genet 2009; 46: 331-337. 
30 Chida A, Shintani M, Nakayama T, et al. Missense mutations of the BMPR1B (ALK6) gene in childhood idiopathic pulmonary arterial hypertension. Circ J 2012; 76: 1501-1508.

31 Chida A, Shintani M, Matsushita Y, et al. Mutations of NOTCH3 in childhood pulmonary arterial hypertension. Mol Genet Genomic Med 2014; 2: 229-239.

32 Yang Y, Chen D, Yuan Z, et al. Megakaryocytic leukemia 1 (MKL1) ties the epigenetic machinery to hypoxia-induced transactivation of endothelin-1. Nucleic Acids Res 2013; 41: 6005-6017.

33 Navas Tejedor P, Tenorio Castaño J, Palomino Doza J, et al. An homozygous mutation in KCNK3 is associated with an aggressive form of hereditary pulmonary arterial hypertension. Clin Genet 2017; 91: 453-457.

34 Navas P, Tenorio J, Quezada CA, et al. Molecular analysis of BMPR2, TBX4, and KCNK3 and genotypephenotype correlations in Spanish patients and families with idiopathic and hereditary pulmonary arterial hypertension. Rev Esp Cardiol 2016; 69: 1011-1019.

35 Levy M, Eyries M, Szezepanski I, et al. Genetic analyses in a cohort of children with pulmonary hypertension. Eur Respir J 2016; 48: 1118-1126.

36 Kerstjens-Frederikse WS, Bongers EM, Roofthooft MT, et al. TBX4 mutations (small patella syndrome) are associated with childhood-onset pulmonary arterial hypertension. J Med Genet 2013; 50: 500-506.

37 Nimmakayalu M, Major H, Sheffield V, et al. Microdeletion of 17q22q23.2 encompassing TBX2 and TBX4 in a patient with congenital microcephaly, thyroid duct cyst, sensorineural hearing loss, and pulmonary hypertension. Am J Med Genet A 2011; 155A: 418-423.

38 Evans JD, Girerd B, Montani D, et al. BMPR2 mutations and survival in pulmonary arterial hypertension: an individual participant data meta-analysis. Lancet Respir Med 2016; 4: 129-137.

39 Ghigna MR, Guignabert C, Montani D, et al. BMPR2 mutation status influences bronchial vascular changes in pulmonary arterial hypertension. Eur Respir J 2016; 48: 1668-1681.

40 Eichstaedt CA, Song J, Viales RR, et al. First identification of Krüppel-like factor 2 mutation in heritable pulmonary arterial hypertension. Clin Sci 2017; 131: 689-698.

41 Song J, Eichstaedt CA, Viales RR, et al. Identification of genetic defects in pulmonary arterial hypertension by a new gene panel diagnostic tool. Clin Sci 2016; 130: 2043-2052.

42 Hadinnapola C, Bleda M, Haimel M, et al. Phenotypic characterization of EIF2AK4 mutation carriers in a large cohort of patients diagnosed clinically with pulmonary arterial hypertension. Circulation 2017; 136: 2022-2033.

43 Hemnes AR, Zhao M, West J, et al. Critical genomic networks and vasoreactive variants in idiopathic pulmonary arterial hypertension. Am J Respir Crit Care Med 2016; 194: 464-475.

44 Rhodes CJ, Wharton J, Ghataorhe P, et al. Plasma proteome analysis in patients with pulmonary arterial hypertension: an observational cohort study. Lancet Respir Med 2017; 5: 717-726.

45 Benza RL, Gomberg-Maitland M, Demarco T, et al. Endothelin-1 pathway polymorphisms and outcomes in pulmonary arterial hypertension. Am J Respir Crit Care Med 2015; 192: 1345-1354.

46 Pencina MJ, Peterson ED. Moving from clinical trials to precision medicine: the role for predictive modeling. JAMA 2016; 315: 1713-1714.

47 Granton J, Langleben D, Kutryk MB, et al. Endothelial NO-synthase gene-enhanced progenitor cell therapy for pulmonary arterial hypertension: the PHACeT Trial. Circ Res 2015; 117: 645-654.

48 Nakhleh MK, Haick H, Humbert M, et al. Volatolomics of breath as an emerging frontier in pulmonary arterial hypertension. Eur Respir J 2017; 49: 1601897.

49 Pollett JB, Benza RL, Murali S, et al. Harvest of pulmonary artery endothelial cells from patients undergoing right heart catheterization. J Heart Lung Transplant 2013; 32: 746-749.

50 Benza RL, Williams G, Wu C, et al. In situ expression of Bcl-2 in pulmonary artery endothelial cells associates with pulmonary arterial hypertension relative to heart failure with preserved ejection fraction. Pulm Circ 2016; 6: 551-556.

51 Kanwar M, Raina A, Passineau M, et al. Idiopathic pulmonary arterial hypertension: evolving therapeutic strategies. Semin Respir Crit Care Med 2017; 38: 606-618.

$52 \mathrm{Gu}$ M, Shao NY, Sa S, et al. Patient-specific iPSC-derived endothelial cells uncover pathways that protect against pulmonary hypertension in BMPR2 mutation carriers. Cell Stem Cell 2017; 20: 490-504.

53 Sa S, Gu M, Chappell J, et al. Induced pluripotent stem cell model of pulmonary arterial hypertension reveals novel gene expression and patient specificity. Am J Respir Crit Care Med 2017; 195: 930-941.

54 Rich S, Brundage BH. High-dose calcium channel-blocking therapy for primary pulmonary hypertension: evidence for long-term reduction in pulmonary arterial pressure and regression of right ventricular hypertrophy. Circulation 1987; 76: 135-141.

55 Sitbon O, Humbert M, Jaïs X, et al. Long-term response to calcium channel blockers in idiopathic pulmonary arterial hypertension. Circulation 2005; 111: 3105-3111.

56 Hemnes AR, Trammell AW, Archer SL, et al. Peripheral blood signature of vasodilator-responsive pulmonary arterial hypertension. Circulation 2015; 131: 401-409.

57 Jais $\mathrm{X}$, Launay $\mathrm{D}$, Yaici $\mathrm{A}$, et al. Immunosuppressive therapy in lupus- and mixed connective tissue disease-associated pulmonary arterial hypertension: a retrospective analysis of twenty-three cases. Arthritis Rheum 2008; 58: 521-531.

58 Degano B, Guillaume M, Savale L, et al. HIV-associated pulmonary arterial hypertension: survival and prognostic factors in the modern therapeutic era. AIDS 2010; 24: 67-75.

59 Savale L, Magnier R, Le Pavec J, et al. Efficacy, safety and pharmacokinetics of bosentan in portopulmonary hypertension. Eur Respir J 2013; 41: 96-103.

60 Soon E, Holmes AM, Treacy CM, et al. Elevated levels of inflammatory cytokines predict survival in idiopathic and familial pulmonary arterial hypertension. Circulation 2010; 122: 920-927.

61 Cracowski JL, Chabot F, Labarère J, et al. Proinflammatory cytokine levels are linked to death in pulmonary arterial hypertension. Eur Respir J 2014; 43: 915-917.

62 Tamby MC, Humbert M, Guilpain P, et al. Antibodies to fibroblasts in idiopathic and scleroderma-associated pulmonary hypertension. Eur Respir J 2006; 28: 799-807.

63 Tamby MC, Chanseaud Y, Humbert M, et al. Anti-endothelial cell antibodies in idiopathic and systemic sclerosis associated pulmonary arterial hypertension. Thorax 2005; 60: 765-772.

64 Rich S, Kieras K, Hart K, et al. Antinuclear antibodies in primary pulmonary hypertension. J Am Coll Cardiol 1986; 8: 1307-1311. 
65 Dib H, Tamby MC, Bussone G, et al. Targets of anti-endothelial cell antibodies in pulmonary hypertension and scleroderma. Eur Respir J 2012; 39: 1405-1414.

66 Tamosiuniene R, Tian W, Dhillon G, et al. Regulatory $\mathrm{T}$ cells limit vascular endothelial injury and prevent pulmonary hypertension. Circ Res 2011; 109: 867-879.

67 Huertas A, Tu L, Gambaryan N, et al. Leptin and regulatory T-lymphocytes in idiopathic pulmonary arterial hypertension. Eur Respir J 2012; 40: 895-904.

68 Hautefort A, Girerd B, Montani D, et al. T-helper 17 cell polarization in pulmonary arterial hypertension. Chest 2015; 147: 1610-1620.

69 Perros F, Dorfmüller P, Souza R, et al. Dendritic cell recruitment in lesions of human and experimental pulmonary hypertension. Eur Respir J 2007; 29: 462-468.

70 Perros F, Dorfmüller P, Montani D, et al. Pulmonary lymphoid neogenesis in idiopathic pulmonary arterial hypertension. Am J Respir Crit Care Med 2012; 185: 311-321.

71 Colvin KL, Cripe PJ, Ivy DD, et al. Bronchus-associated lymphoid tissue in pulmonary hypertension produces pathologic autoantibodies. Am J Respir Crit Care Med 2013; 188: 1126-1136.

72 Kawut SM, Bagiella E, Lederer DJ, et al. Randomized clinical trial of aspirin and simvastatin for pulmonary arterial hypertension: ASA-STAT. Circulation 2011; 123: 2985-2993.

73 Lindemann S, Gierer C, Darius H. Prostacyclin inhibits adhesion of polymorphonuclear leukocytes to human vascular endothelial cells due to adhesion molecule independent regulatory mechanisms. Basic Res Cardiol 2003; 98: 8-15.

74 Shinomiya S, Naraba $\mathrm{H}$, Ueno A, et al. Regulation of TNF $\alpha$ and interleukin-10 production by prostaglandins $\mathrm{I}_{2}$ and $\mathrm{E}_{2}$ : studies with prostaglandin receptor-deficient mice and prostaglandin E-receptor subtype-selective synthetic agonists. Biochem Pharmacol 2001; 61: 1153-1160.

75 Wang JW, Vu C, Poloso NJ. A prostacyclin analog, cicaprost, exhibits potent anti-inflammatory activity in human primary immune cells and a uveitis model. J Ocul Pharmacol Ther 2017; 33: 186-192.

76 Rosenzweig EB, Morse JH, Knowles JA, et al. Clinical implications of determining BMPR2 mutation status in a large cohort of children and adults with pulmonary arterial hypertension. J Heart Lung Transplant 2008; 27: 668-674.

77 Trip P, Girerd B, Bogaard HJ, et al. Diffusion capacity and BMPR2 mutations in pulmonary arterial hypertension. Eur Respir J 2014; 43: 1195-1198.

78 Stacher E, Graham BB, Hunt JM, et al. Modern age pathology of pulmonary arterial hypertension. Am J Respir Crit Care Med 2012; 186: 261-272.

79 Ghigna MR, Hamdi S, Petitpretz P, et al. Angiomatoid fibrous histiocytoma of the pulmonary artery: a multidisciplinary discussion. Histopathology 2014; 65: 278-282.

80 Guignabert C, Bailly S, Humbert M. Restoring BMPRII functions in pulmonary arterial hypertension: opportunities, challenges and limitations. Expert Opin Ther Targets 2017; 21: 181-190.

81 Girerd B, Weatherald J, Montani D, et al. Heritable pulmonary hypertension: from bench to bedside. Eur Respir Rev 2017; 26: 170037.

82 Eyries M, Montani D, Girerd B, et al. EIF2AK4 mutations cause pulmonary veno-occlusive disease, a recessive form of pulmonary hypertension. Nat Genet 2014; 46: 65-69.

83 Atkinson C, Stewart S, Upton PD, et al. Primary pulmonary hypertension is associated with reduced pulmonary vascular expression of type II bone morphogenetic protein receptor. Circulation 2002; 105: 1672-1678.

84 Reynolds AM, Holmes MD, Danilov SM, et al. Targeted gene delivery of BMPR2 attenuates pulmonary hypertension. Eur Respir J 2012; 39: 329-343.

85 Harper RL, Reynolds AM, Bonder CS, et al. BMPR2 gene therapy for PAH acts via Smad and non-Smad signalling. Respirology 2016; 21: 727-733.

86 Yan L, Chen X, Talati M, et al. Bone marrow-derived cells contribute to the pathogenesis of pulmonary arterial hypertension. Am J Respir Crit Care Med 2016; 193: 898-909.

87 Spiekerkoetter E, Tian X, Cai J, et al. FK506 activates BMPR2, rescues endothelial dysfunction, and reverses pulmonary hypertension. J Clin Invest 2013; 123: 3600-3613.

88 Spiekerkoetter E, Sung YK, Sudheendra D, et al. Low-dose FK506 (tacrolimus) in end-stage pulmonary arteria hypertension. Am J Respir Crit Care Med 2015; 192: 254-257.

89 Spiekerkoetter E, Sung YK, Sudheendra D, et al. Randomised placebo-controlled safety and tolerability trial of FK506 (tacrolimus) for pulmonary arterial hypertension. Eur Respir J 2017; 50: 1602249.

90 Long L, Yang X, Southwood M, et al. Chloroquine prevents progression of experimental pulmonary hypertension via inhibition of autophagy and lysosomal bone morphogenetic protein type II receptor degradation. Circ Res 2013; 112: 1159-1170.

91 Dunmore BJ, Drake KM, Upton PD, et al. The lysosomal inhibitor, chloroquine, increases cell surface BMPR-II levels and restores BMP9 signalling in endothelial cells harbouring BMPR-II mutations. Hum Mol Genet 2013; 22 : 3667-3679. 